Relations industrielles

Industrial Relations

\title{
Man at Work, by William Foote Whyte, Homewood, Illinois, R.D. Irwin, Inc., 1961, pp. 593, \$10.60.
}

\section{Louis-Marie Tremblay}

Volume 16, numéro 4, octobre 1961

URI : https://id.erudit.org/iderudit/1021701ar

DOI : https://doi.org/10.7202/1021701ar

Aller au sommaire du numéro

Éditeur(s)

Département des relations industrielles de l’Université Laval

ISSN

0034-379X (imprimé)

1703-8138 (numérique)

Découvrir la revue

Citer ce compte rendu

Tremblay, L.-M. (1961). Compte rendu de [Man at Work, by William Foote Whyte, Homewood, Illinois, R.D. Irwin, Inc., 1961, pp. 593, \$10.60.] Relations industrielles / Industrial Relations, 16(4), 514-515.

https://doi.org/10.7202/1021701ar

Tous droits réservés @ C Département des relations industrielles de l’Université Laval, 1961
Ce document est protégé par la loi sur le droit d'auteur. L'utilisation des services d'Érudit (y compris la reproduction) est assujettie à sa politique d'utilisation que vous pouvez consulter en ligne.

https://apropos.erudit.org/fr/usagers/politique-dutilisation/ 
sous prétexte de maintenir la paix sociale et de protéger le bien commun. La partie V analyse le rôle du gouvernement dans la détermination des salaires et des heures de travail. Il y a une bonne revue historique de la règlementation statutaire des salaires: lois des salaires minimums, standards des justes salaires, stabilisation employée pendant la deuxième guerre mondiale; ainsi que leur portée et leurs effets. Une heureuse attention est aussi accordée aux aspects économiques ainsi qu'aux facteurs qui affectent la réduction des heures de travail. Dans la partie VII on fait une revue des principales règlementations gouvernementales concernant le syndicalisme. Sur ce, un chapitre porte sur le contrôle des tactiques syndicales, grève, piquetage, etc. Puis, il y a des analyses très élaborées du \& TaftHartley Act » et du \& Landrum-Griffin Act précédées d'une étude malheureusement insuffisante et inadéquate du Wagner Act. Cette partie se termine avec le rôle du gouvernement comme conciliateur dans les conflits industriels. Finalement, la dernière partie s'efforce de souligner les principaux problèmes internes et externes que le mouvement syndical rencontre en 1960.

Donc, voici un manuel remis à neuf qui tient compte non seulement des aspects économiques mais qui accorde aussi une large part aux aspects historiques et règlementaires, pour s'avérer lun des plus complets et des mieux construits dans le domaine.

\section{Louts-Marue Tremblay}

Man at Work, by William Foote Whyte, Homewood, Illinois, R.D. Irwin, Inc., 1961 , pp. 593, $\$ 10.60$.

L'auteur de «Industry and Society 》 et de * Human Relations in the Restaurant Industry » nous livre maintenant la somme de vingt ans d'expérience en matière de relations humaines dans le milieu du travail. «Man at Work »'impose immédiatement comme l'un des meilleurs volumes dans le domaine. A l'encontre de la plupart des « textbooks 》 qui consiste surtout dans un amas de matériel descriptif, «Man at Work 》 présente un heureux alliage entre la description, les cas et l'analyse théorique. L'auteur procède par induction, dans une approche qui concilie et se situe entre les approches traditionnelles adop- tées par les psychologues et les sociologues.

La première partie sert à élaborer les instruments d'analyse. Whyte s'interroge d'abord sur les déterminants des inter-relations sociales dans le milieu du travail. Pour y répondre, il adopte la méthode dite: «théorie de l'interaction », développée initialement par Arensberg et McGregor, méthode basée sur les concepts: sentiments, activités et interactions. Ces derniers sont les résultantes a) des influences sociales et culturelles qui moulent la personnalité, b) des forces impersonnelles qui agissent sur le travailleur dans le milieu du travail, c) des interactions et des activités des autres. Ceci constitue l'essentiel du schème analytique que l'auteur utilise dans les parties subséquentes.

Les parties 2 et 3 analysent les influences respectives de l'environnement socio-économique et de l'environnement technique et physique sur le schème trinaire, plus spécialement sur la formation des sentiments. Les parties 4,5 et 6 traitent de l'organisation formelle. La partie 4 étudie intensivement les relations patronales ouvrières. La partie 5 porte sur les problèmes qui concernent la hiérarchie (le « line 》) et la partie 6 sur ceux reliés aux différents services («staffs »). Tout au cours de ces développements, l'auteur cherche à dégager les facteurs d'intégration des travailleurs dans leur milieu tant verticalement qu'horizontalement en utilisant largement le cas pour fin d'illustration. Toutefois, l'auteur sous-estime l'importance de l'organisation non-formelle à laquelle il ne consacre d'ailleurs aucun chapitre particulier. De même, le traitement intégral de l'aspect analytique des concepts n'entre pas dans les préoccupations de l'auteur. C'est pourquoi, « Man at Work » nous apparaît comme un complément idéal à un volume de caractère analytique comme * Human Relations in Administration 》 de Robert Dubin.

La dernière partie qui vise à une reformulation théorique par rapport à l'individu, au groupe et à l'organisation, sert de pendant à la première partie. Ce n'est pas une théorie en soi. Mais l'auteur pose les premiers jalons vers un effort futur de théorisation plus complète, laquelle sera rendue possible par l'augmentation de notre connaissance et 
de notre compréhension de l'homme au travail.

Il est de notre opinion que « Man at Work s'avèrera un instrument de travail très utile tant pour les milieux industriels que pour les milieux académiques.

\section{Louis-Marie Tremblay}

Trade Union Development and Industrial Relations in the British West Indies, par William S. Knowles. A publication of the Institute of Industrial Relations, University of California. University of California Press, Berkeley and Los Angeles, 1959. 214 pages.

Les Indes occidentales britanniques sont constituées d'un ensemble d'îles dans la mer des Caraïbes et de deux pays reliés au continent: les Guyannes anglaises et le Honduras britannique. La plupart de ces colonies se sont groupées, en 1958, dans une fédération. Chacune possède son économje propre, son histoire. Mais ces pays ont de commun qu'ils sont sous-développés et évoluent à l'intérieur d'institutions britanniques. Le mouvement syndical y est d'origine récente. Il remonte seulement à 1939 . Ainsi l'évolution politique vers un statut de gouvernement autonome se place historiquement au même moment que la naissance et la croissance du syndicalisme.

L'auteur s'est proposé d'analyser le développement du syndicalisme en rapport avec les conditions économiques, sociales et politiques. Pour faire son tra- vail, il a d'abord vécu dans l'une de ces îles pendant un an, il a parcouru toute la littérature existente et, en 1953-54, il a passé quatorze mois à interviewer les propriétaires fonciers, les secrétaires des associations d'employeur, les gérants d'exploitations, les administrateurs des colonies, les dirigeants syndicaux et les politiciens.

L'auteur est d'une grande probité tant dans les méthodes qu'il a utilisées que dans les, conclusions qu'il tire de son analyse. Après avoir exposé l'arrièreplan général de ces colonies, les récents développements économiques, sociaux, culturels et politiques, il donne un tableau du syndicalisme ouvrier. Puis il montre les facteurs externes qui ont influencé le développement du syndicalisme. Dans chacun des pays, il analyse les rapports entre le syndicalisme ouvrier et les partis politiques, l'état des relations patronales-ouvrières et l'attitude des syndicats en face des problèmes économiques fondamentaux.

Bien que le syndicalisme soit grandement influencé dans ses structures, son orientation, son action par les conditions du milieu, comme cette monographie le démontre, il est possible de retrouver une évolution analogue dans tous les pays en voie de développement. Cet ouvrage est intéressant et utile pour tous ceux qui recherchent la vraie nature du syndicalisme. Il est une contribution importante à l'étude comparée du syndicalisme ouvrier dans le monde.

\section{GÉrard Dion}

\section{PUBLICATIONS RÉCENTES RECENT PUBLICATIONS}

\section{Généralités}

« L'Eglise et les structures de la vie sociale moderne », par Mgr E. Dejardin, Les Dossiers de l'Action sociale catholique, 38e année, no 5, mai-juin 1961, pp. $331-341$.

* The American Writer Views the American Businessman », by Royall Brandis, The Quarterly Review of Economics and
Business, Vol. I, No. 3, August 1961, pp. 29-38.

«Industrial Survival in Nuclear War », by D.V.T. Bear and P.G. Clark, The Quarterly Review of Economics and Business, Vol. I, No. 3, August 1961. pp. 39-52.

« Organization Theory: A Survey of Three Views », by Charles Z. Wilson, 